\title{
A Virus-Like Particle-Based Vaccine Candidate against the Tick-Borne Powassan Virus Induces Neutralizing Antibodies in a Mouse Model
}

\author{
Velasco Cimica *(D), Sahar Saleem, Emily Matuczinski, Debra Adams-Fish, Conor McMahon, Sujatha Rashid \\ and Timothy T. Stedman $\mathbb{D}$
}

check for updates

Citation: Cimica, V.; Saleem, S.; Matuczinski, E.; Adams-Fish, D.; McMahon, C.; Rashid, S.; Stedman, T.T. A Virus-Like Particle-Based Vaccine Candidate against the Tick-Borne Powassan Virus Induces Neutralizing Antibodies in a Mouse Model. Pathogens 2021, 10, 680. https://doi.org/10.3390/ pathogens 10060680

Academic Editor: Anna Honko

Received: 7 April 2021

Accepted: 25 May 2021

Published: 31 May 2021

Publisher's Note: MDPI stays neutral with regard to jurisdictional claims in published maps and institutional affiliations.

Copyright: (C) 2021 by the authors Licensee MDPI, Basel, Switzerland. This article is an open access article distributed under the terms and conditions of the Creative Commons Attribution (CC BY) license (https:// creativecommons.org/licenses/by/ $4.0 /)$.
American Type Culture Collection (ATCC®), ATCC Federal Solutions, 10801 University Boulevard, Manassas, VA 20110, USA; ssaleem2@gmu.edu (S.S.); e.matuczinski@yahoo.com (E.M.);

DAdams-Fish@atcc.org (D.A.-F.); cmcmahon@atcc.org (C.M.); srashid@atcc.org (S.R.); TStedman@atcc.org (T.T.S.)

* Correspondence: velascodoc@aol.com

\begin{abstract}
Powassan virus (POWV) is a tick-borne flavivirus circulating in North America and the Russian Far East that can cause severe neuroinvasive diseases, including encephalitis, meningitis, and meningoencephalitis. The reported neuroinvasive case fatality is about $10 \%$, and approximately $50 \%$ of the survivors from the neuroinfection exhibit long-lasting or permanent neurological sequelae. Currently, treatment of POWV infection is supportive, and no FDA-approved vaccines or specific therapeutics are available. A novel Powassan vaccine candidate was created using virus-like particle technology (POW-VLP) and assembled with the viral structural proteins pre-Membrane (prM) and Envelope (E). Western blot immunoassay demonstrated high antigenicity of POW-VLP structural proteins. Transmission electron microscopy indicated that the POW-VLP exhibited icosahedral morphology typical of flaviviruses. A dose-escalation study in a murine model was performed to test immunogenicity and safety. Serum antibody was tested by ELISA, demonstrating that POWVLP afforded $100 \%$ seroconversion to the E protein. Reporter viral-particle neutralization assay demonstrated high levels of neutralizing antibodies in the serum of immunized mice. Hybridomas expressing monoclonal antibodies were produced following POW-VLP immunization. The POWVLP vaccine candidate created in this study provides a strategy for inducing protective antibodies against Powassan neuroinvasive infection.
\end{abstract}

Keywords: Powassan virus; vaccine; virus-like particle; POWV; VLP; flavivirus

\section{Introduction}

Powassan virus (POWV) is a tick-borne flavivirus pathogen that was first discovered and isolated in Ontario, Canada, in 1958 [1]. POWV infections in humans are sporadic in North America and the Russian Far East [2-4]. The viral disease may initiate with flu-like symptoms, but about half of reported cases result in severe neuroinvasive disease, including encephalitis, meningitis, and meningoencephalitis. The fatality rate of neuroinvasive POWV cases is approximately $12 \%$, and approximately $50 \%$ of neuroinvasive infections result in permanent brain damage associated with long-lasting neurological sequelae such as headaches, muscle weakness, focal paralysis, or cognitive difficulties [5]. Currently, treatment of POWV infection is supportive, and no FDA-approved vaccines or specific therapeutics are available.

In the USA, the incidence of POWV disease reported to the Centers for Disease Control and Prevention (CDC) has increased over the last two decades from about one case per year before 2005 to an average of more than 24 domestic cases annually since 2016 [6]. Importantly, a record of 39 cases was reported in 2019. Recent evidence suggests POWV is transmissible through blood transfusion, creating a risk for transmission through the blood supply [7]. POWV is characterized by two lineages serologically indistinguishable 
but circulating with a specific tick vector: lineage I is typically vectored by Ixodes cookei, while lineage II, also called deer tick virus (DTV), is usually transmitted by Ixodes scapularis. The emergence of POWV cases may have resulted from the virus lineage adaptation to the more aggressive tick species $I x$. scapularis from the $I x$. cookei that rarely bites humans. Indeed, Ix. scapularis has geographically expanded in recent decades across much of the eastern and central US [4] and is a primary vector for Lyme disease and the lineage II of POWV [8]. It is speculated that the combined effects of reforestation, urban sprawl, and greater human population density near the vector habitat [8] have driven increases in tick-borne diseases. Coincident with an increase in POWV infections, multiple states have reported their first confirmed cases in the last 5 years $[9,10]$. Given the increase in POWV incidence and expansion, human urbanization of rural areas, increase in the tick population, lack of therapeutics, and the potentially morbid consequences of POWV infection, proactive development of a POWV vaccine should be a public health priority.

Several VLP vaccine candidates have been developed employing the E protein as antigen [11,12]. Flavivirus E protein comprises the majority of the virion structure and surface [13]. The E protein plays multiple roles in the viral infection cycle, including host receptor recognition and binding, membrane fusion with the cell, virion maturation, assembly, and egress from the cell [14]. Indeed, most flavivirus vaccines deliver the E protein as an immunogen due to the induction of virus-neutralizing and protective antibodies.

The flavivirus E protein has a conserved structure divided into three domains. These include the amino-terminal domain I (EDI) consisting of a beta-barrel within the middle of the protein monomer; domain II (EDII), which includes a fusion peptide that is functionally important for dimerization and virion assembly; and the carboxy-terminal domain III (EDIII), which is characterized by an immunoglobulin-like domain. Multiple studies have demonstrated that EDIII is the ideal antigen for eliciting neutralizing and protective antibodies against both tick-borne [15,16] and mosquito-borne flaviviruses [17,18].

Herein, we report the development of the first virus-like particle (VLP)-based vaccine candidate against POWV. VLP vaccines are based on the self-assembly of viral proteins into a structure resembling the native virion. The morphology of VLPs enhances epitope presentation and immune stimulation for both humoral and cellular responses [19]. VLPs exhibit high safety profiles because they lack the genetic material required for viral replication [20]. We produced Powassan VLPs (POW-VLP) in mammalian cells using the prM-E structural polyprotein. The POW-VLP exhibited the structural morphology of icosahedral virions. The immunization of mice with POW-VLPs induced neutralizing antibodies, and monoclonal antibodies targeted to POWV E protein were generated.

\section{Results}

\subsection{Characterization of POWV EDIII Recombinant Protein}

A truncated POWV E protein encompassing domain III (EDIII) was engineered as a control antigen. The POWV EDIII was expressed with a C-terminal histidine tag for detection and purification by immobilized metal affinity chromatography (IMAC). The recombinant protein was produced in a yeast system (Pichia pastoris) with a high yield of expression. SDS-PAGE and Coomassie blue staining analysis showed over $95 \%$ purity of POWV EDIII (Figure 1, panel 1), with electrophoretic mobility in agreement with the predicted molecular weight of $12.7 \mathrm{kDa}$ obtained by bioinformatics analysis [21]. Western blotting analysis showed POWV EDIII reactivity using anti-histidine antibody and aligning with a similar molecular size and reactivity with respect to a Zika Virus (ZIKV) EDIII histidine-tag recombinant protein standard (Figure 1, panel 2). In addition, POWV EDIII reacted specifically with anti-POWV ascitic fluid antibody (POWV AF) (Figure 1, panel 3). The monoclonal antibody against E protein from the closely related Langat virus (LGTV) that shares the same serocomplex with POWV [22] was cross-reactive with the POWV EDIII recombinant protein (Figure 1, panel 4). Neither POWV AF nor LGTV E antibodies crossreacted with the more divergent ZIKV EDIII recombinant protein (Figure 1, panels 3, 4). 


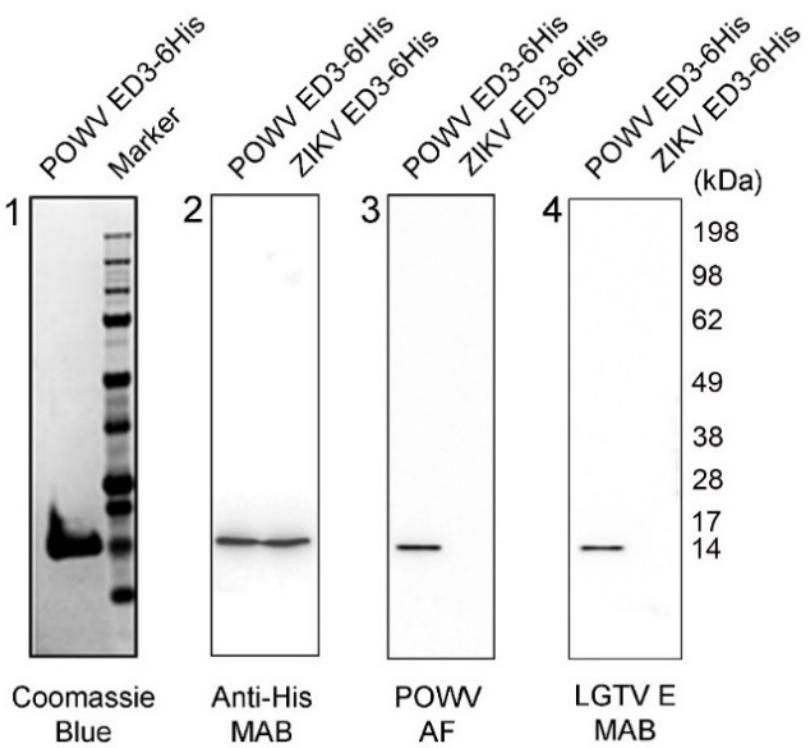

Figure 1. Analysis of POWV EDIII recombinant protein by SDS-PAGE and Western blot. Coomassie blue staining indicated electrophoretic mobility of approximately $12.7 \mathrm{kDa}$ (left panel 1). Western blot analysis of POWV EDIII and ZIKV EDIII control (panels 2 to 4 from left). The anti-histidine monoclonal antibody (Anti-His MAB) detected both recombinant proteins (panel 2). Anti-POWV ascitic fluid antibody (POWV AF) (panel 3) and LGTV anti-envelope protein monoclonal antibody (LGTV E MAB) (panel 4) reacted specifically with POWV EDIII and not with ZIKV EDIII.

\subsection{Production of POW-VLPs in a Mammalian System}

An expression construct encoding the polyprotein truncation prM-E was engineered for the expression of POW-VLP in mammalian cells (Figure 2a). The POW-VLP construct was transfected into 293T cells, and POWV E expression was confirmed by indirect immunofluorescence with a POWV ascitic fluid antibody. POWV E protein was abundant in the extranuclear compartment of the transfected cells as expected (Figure 2b). Furthermore, Western blot analysis demonstrated that POW-VLP was poorly secreted in the culture media and accumulated in the cytosolic fraction (Figure 2c). The bulk of the POWV E protein migrated to approximately $54 \mathrm{kDa}$ as predicted for the mature protein in agreement with the literature and bioinformatics analysis [21,23], with a minor band present at an apparent molecular weight of approximately $60 \mathrm{kDa}$, which may represent the uncleaved M-E protein.

\subsection{POW-VLPs Demonstrate High Antigenicity and Virion Morphology}

Purified POW-VLP were characterized for antigenicity using multiple monoclonal antibodies. POWV E protein was detected with both POWV ascitic fluid and LGTV anti-E protein monoclonal antibodies (Figure 3A). Both antibodies detected a major band with a molecular weight of approximately $54 \mathrm{kDa}$ (Figure 3A, panels 1 and 2). The prM protein was detected with another monoclonal antibody presenting a band with a molecular size of approximately $18 \mathrm{kDa}$ (Figure 3A, panel 3). Virion morphology of POW-VLPs was examined by transmission electron microscopy (TEM) and negative staining. The VLPs exhibited icosahedral morphology typical of flavivirus and sizes between 40 and $50 \mathrm{~nm}$, in agreement with previous studies regarding POWV [24,25].

\subsection{POW-VLPs Elicit Antibody Response against POWV EDIII Protein}

POW-VLPs were produced and purified at a large scale in mammalian cells as described above. The concentration of POW-VLP was measured by dot blot immunoassay using a POWV ascitic fluid antibody and POWV-EDII recombinant protein as a molec- 
ular standard. The POW-VLP immunization dose was based on the quantity of EDIII antigen delivered.

\section{A}

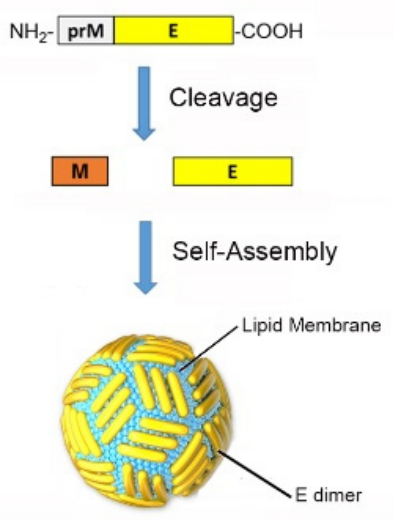

B

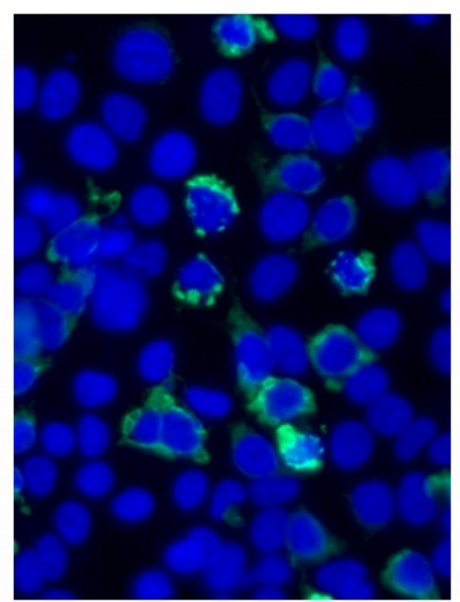

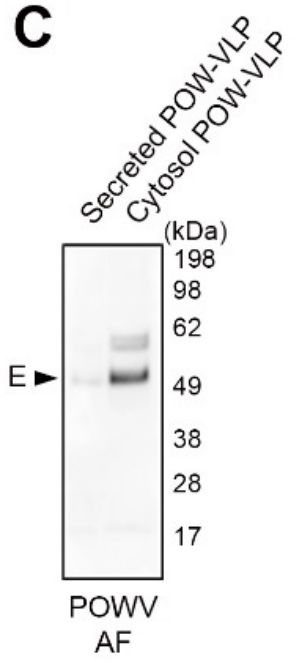

Figure 2. Production of POW-VLP in mammalian cells. (A) The expressed recombinant E protein used for POW-VLP generation undergoes processing and self-assembly into virus-like particles. (B) Indirect immunofluorescence detection of the POWV E protein expressed in transfected mammalian cells. (C) Purified POW-VLPs were detected by Western blot analysis from cell culture supernatants and the cytosolic extract of transfected mammalian cells. POW-VLPs were poorly secreted and were primarily retained in the cytosol. Both assays were performed using anti-POWV ascitic fluid antibodies.

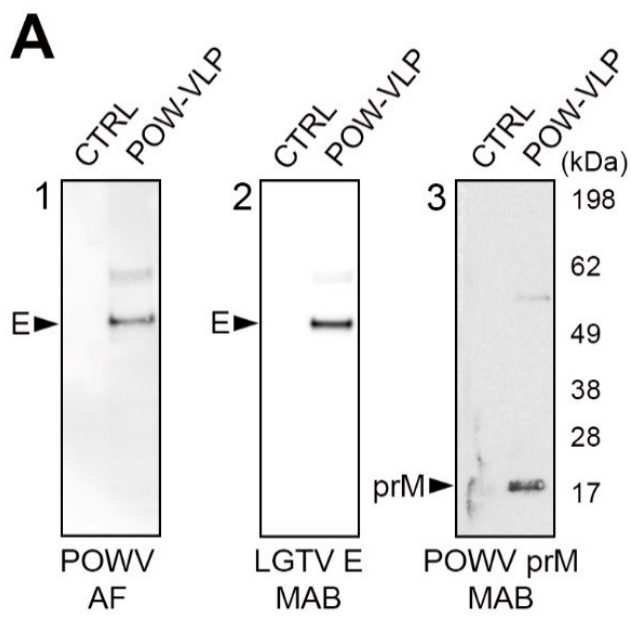

B
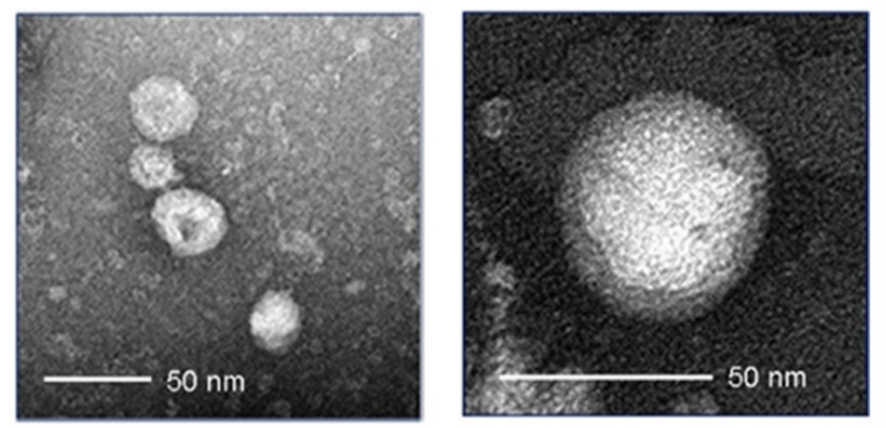

Figure 3. Characterization of POW-VLPs. (A) POW-VLP antigenicity assessed by Western blot analysis. Anti-POWV ascitic fluid antibodies (POWV AF) (panel 1) and LGTV anti-envelope protein monoclonal antibodies (LGTV E MAB) (panel 2) recognized E protein from POW-VLP; the presence of prM protein from POW-VLPs was detected using POWV anti-prM antibody (panel 3). (B) Transmission electron microscopy analysis indicated icosahedral morphology and size of 40-50 $\mathrm{nm}$ for POW-VLP.

A dose-escalation study for POW-VLP was performed in BALB/c mice. The immunization of 10 mice per group ( 5 male, 5 female) was completed by intramuscular injection using a prime and 2 boosts immunizations regimen separated by 3 -week intervals. Mice were dosed with 1,5 , or $10 \mu \mathrm{g}$ of POW-VLP containing EDIII equivalent antigen. In order to increase vaccine efficacy, POW-VLPs were formulated with an adjuvant composed of a squalene-based oil-in-water nano-emulsion (AddaVax).

Mice were observed daily during the entire study, and no adverse effects were found from the immunization protocol, demonstrating the vaccine tolerability. 
Serum samples were collected 3 weeks after prime, first boost, and second boost immunizations and analyzed by ELISA with POWV EDIII recombinant protein as a coating antigen (Figure $4 \mathrm{~A}$ ). The prime immunization resulted in roughly $70 \%$ seroconversion in the immunized mice with no statistical difference in the serum titer between groups (Figure 4A). The serum titer increased after the first boost immunization, reaching a complete seroconversion for the highest dose of $10 \mu \mathrm{g}$ of POW-VLP. The second boost immunization further augmented the antibody response and elicited complete seroconversion from the lowest to the highest dose of POW-VLP. After the final immunization, the serum antibody response was found to be dose-dependent.

A
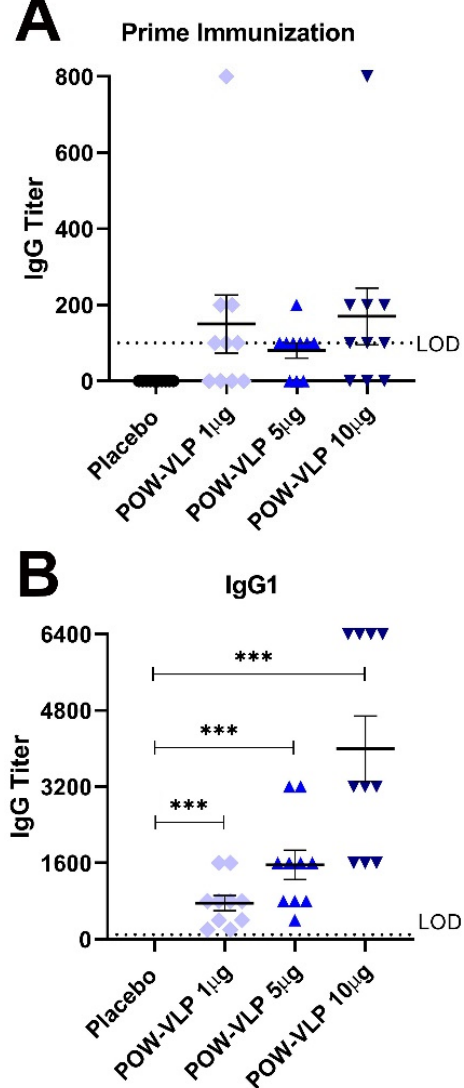
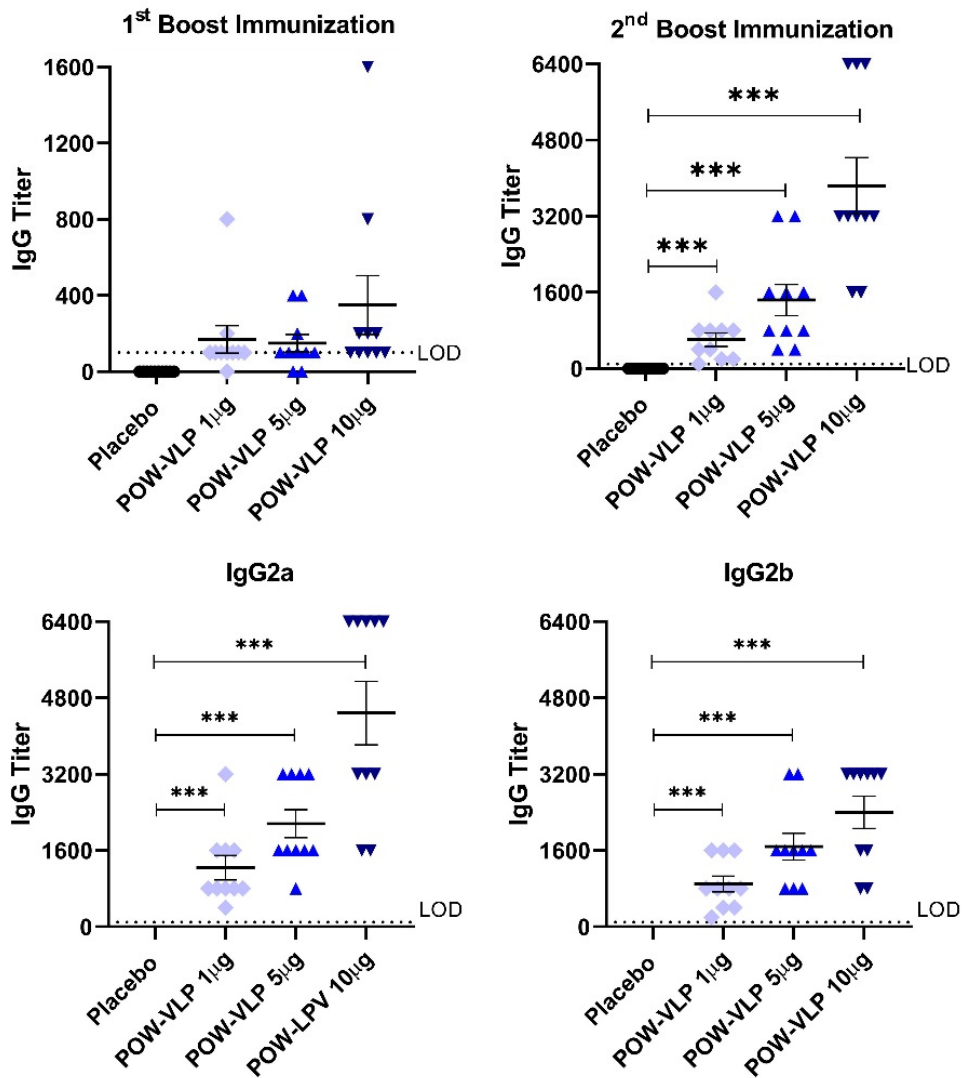

Figure 4. Testing of POW-VLP immunogenicity by ELISA. (A) POW-VLPs induced IgG against POWV EDIII antigen after prime immunization. IgG titer increased after boost immunizations, showing $100 \%$ seroconversion in each dosage group (*** $p<0.0005)$. (B) POW-VLP elicited both $\operatorname{IgG}_{1}$ and $\operatorname{IgG}_{2}(\mathbf{A}, \mathbf{B})$ antibody response after second boost immunization $\left({ }^{* * *} p<0.0005\right)$.

The IgG isotype antibody response was analyzed by ELISA of serum samples collected after the final immunization (second boost) (Figure 4B). POW-VLPs elicited both $\operatorname{IgG}_{1}$ and $\mathrm{IgG}_{2}(\mathrm{a}$ and $\mathrm{b})$, corresponding to Th2- and Th1-immune responses, respectively.

\subsection{POW-VLP Induces Neutralizing Antibodies}

Pooled serum samples collected 3 weeks after the second boost immunization of POW-VLPs were analyzed for neutralization activity using a reporter virus-like particle neutralization test established by Pierson et al. [26]. POW-VLPs elicited a high level of neutralizing antibodies in a dose-dependent manner for each of the 3 dosages of the POWVLP vaccine (1,5, and $10 \mu \mathrm{g}$ of EDIII protein equivalent antigen) (Figure 5). Each dosage group demonstrated statistically significant neutralization activity with respect to placebo control. The high dose of $10 \mu \mathrm{g}$ of POW-VLP elicited antibodies with neutralization activity comparable to anti-POWV ascitic fluid (AF) antibody-positive control. 


\section{POW-VLP Neutralization}

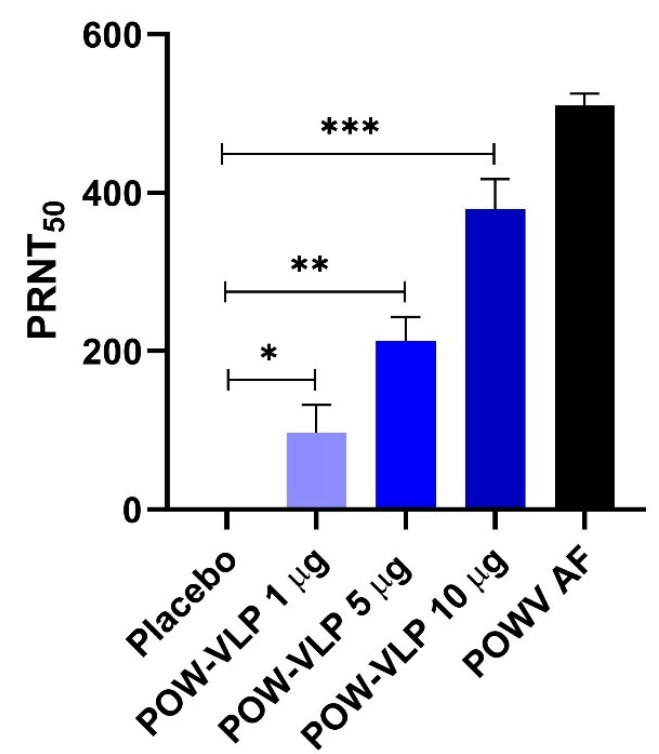

Figure 5. Neutralization test for POW-VLP immunization in a murine model. The reporter virusparticle neutralization test demonstrated that POW-VLPs induce a dose-dependent neutralization activity in a murine model $\left({ }^{*} p<0.05 ;{ }^{* *} p<0.005 ; * * *<0.0005\right)$. Neutralization activity quantification is indicated by $50 \%$ inhibition of plaque reduction neutralization test $\left(\mathrm{PRNT}_{50}\right)$. Anti-POWV ascitic fluid antibody was included as a control (POWV AF).

\subsection{Generation of Monoclonal Antibodies against POWV}

Splenocytes were harvested from a mouse immunized with a high dosage of POWVLP (10 $\mu \mathrm{g}$ EDIII equivalent antigen), and hybridoma clones were isolated for monoclonal antibody production. Hybridoma clones were screened by ELISA using POWV EDIII as a coating antigen. Twenty positive clones were assessed for reactivity by ELISA screening (Figure 6).

IgG Level

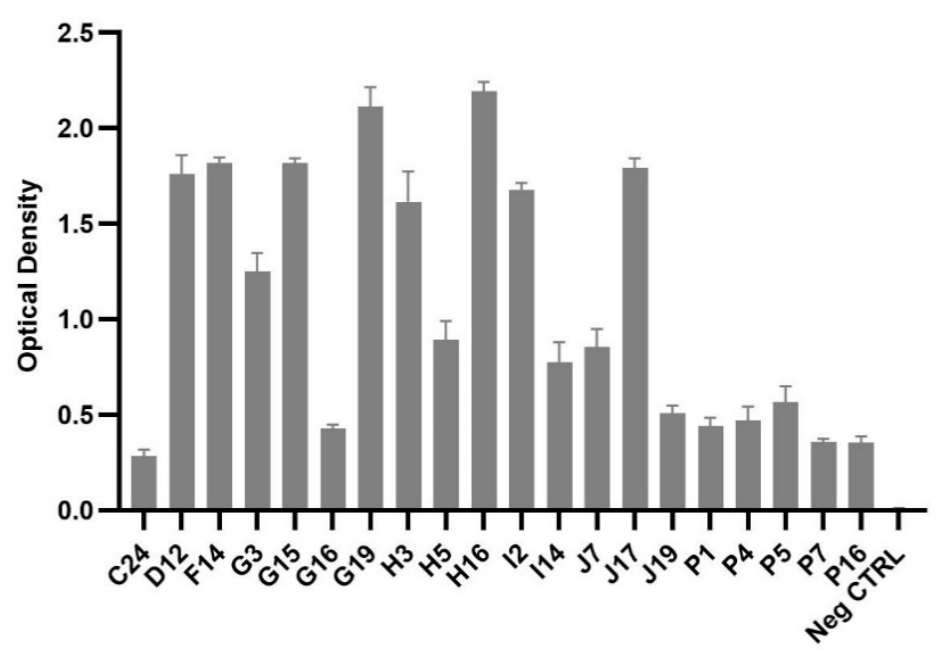

Monoclonal Antibodies

Figure 6. ELISA screening for monoclonal antibodies. Twenty hybridomas expressing monoclonal antibodies were screened for secretion of IgG against POW-EDIII recombinant protein antigen. Optical density values were subtracted against the background control value. 
Hybridoma clones with varying levels of reactivity to POW-EDIII were selected for further screening. Hybridoma supernatants were tested against the POW-VLP antigen by dot blot immunoassay (Figure 7). The dot blot analysis correlated with the ELISA screening showed above (Figures 6 and 7), demonstrating monoclonals with high (Figure 7A), medium (Figure 7B), and low reactivity (Figure 7C).

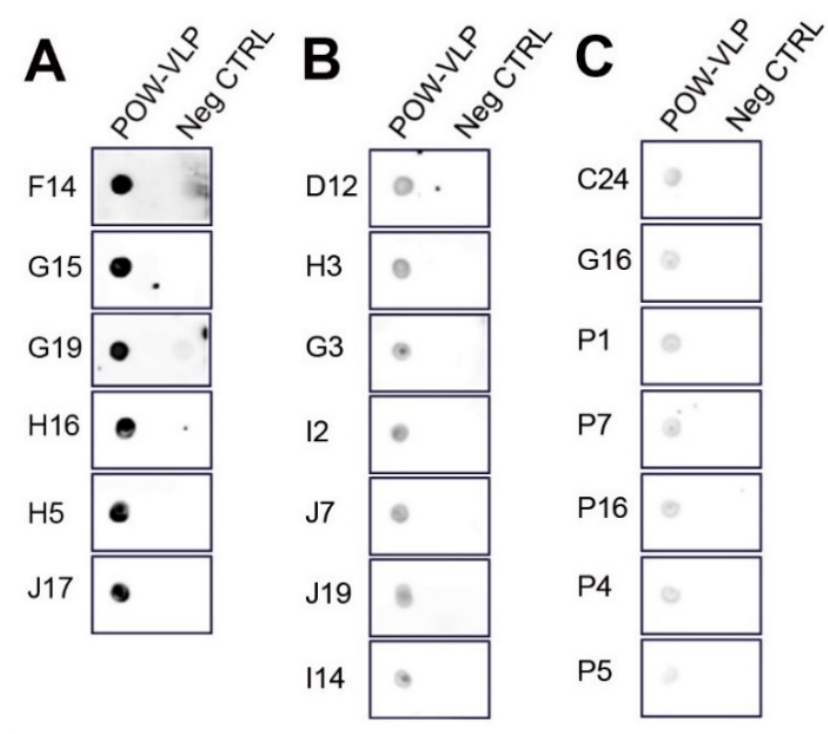

Figure 7. Hybridoma testing by dot blot immunoassay. Supernatant from the hybridoma culture was tested for antibody reactivity against the POW-VLP antigen. Hybridomas were classified as (A) high, (B) medium, and (C) low reactivity. Protein lysate was used as a negative control.

\section{Discussion}

Due to the success of the VLP platform, multiple flavivirus vaccine candidates have been developed. The ability of flavivirus prM-E or C-prM-E polyprotein to self-assemble into an enveloped VLP with similar morphology to the wild-type virus has been harnessed to create highly immunogenic recombinant vaccines $[27,28]$.

We have developed a novel VLP-based vaccine candidate against the tick-borne POWV. To date, only two nucleic acid-based vaccine candidates have been developed against Powassan infection, each exhibiting some efficacy and safety [29,30]. However, RNA-based vaccines require stringent cold chain maintenance for storage and distribution, whereas VLPs are quite stable under standard refrigeration, potentially facilitating the distribution. A VLP-based vaccine presents conformational epitopes from the virion structure that stimulate highly neutralizing and protective antibodies [19,20].

The prM-E based VLPs are efficiently secreted from mammalian and insect cells into the culture media, making this approach convenient for the manufacturing process. While the POW-VLP vaccine candidate was produced robustly in mammalian cells using virus prM-E structural proteins, the expressed POW-VLPs remained predominantly in the intracellular compartment, with low secretion into the culture supernatant. The low degree of secretion suggests that POWV requires the capsid (C) and non-structural (NS) proteins for complete virion maturation, efficient budding, and release from the cell [31,32].

Two recent reports demonstrated that the EDIII antigen from POWV or TBEV elicits broadly neutralizing and protective antibodies against tick-borne flaviviruses. Monoclonals antibodies developed against POWV EDII or EDIII inhibited post-attachment viral entry in the cell [33]. A subset of cross-reacting monoclonal antibodies protected against POWV, Langat virus (LGTV), and TBEV infection in a mouse model. In another study, broadly neutralizing antibodies against tick-borne flavivirus were discovered in humans after TBEV infection [34]. The antibodies were able to bind the TBEV EDIII domain and exert broad neutralization activity against multiple tick-borne flaviviruses, including POWV. 
One isolated monoclonal antibody demonstrated high protection against TBEV infection in a mouse model.

The POW-VLP vaccine candidate produced in this study exhibited high antigenicity and elicited serogroup-specific antibodies against POWV EDIII (Figures 1 and 3A). Using ELISA, we measured the serum antibody response specific to EDIII induced by POW-VLP. However, since POW-VLP expresses the full E protein and epitopes spanning EDI, EDII, prM, and VLP structural antigens, the current analyses may underestimate the total IgG titer against the virus. Hence, the POW-VLP vaccine candidate may induce additional protective antibodies from other epitopes.

The structural assembly of the VLP ensures the conformationally correct presentation of EDIII epitopes to the host immune system. The external shell of a mature flavivirus virion is composed of 180 copies of the E protein arranged in head-to-tail dimers organized on the icosahedral surface of the virion [35]. Transmission electron microscopy analysis demonstrated that POW-VLPs are assembled with icosahedral morphology and size comparable to the native virus (Figure 3B) [24].

In vivo studies demonstrated the POW-VLP stimulated neutralizing antibodies specific for the EDIII domain (Figures 4 and 5). We have used these preliminary studies to produce monoclonal antibodies against POWV. We isolated 20 hybridoma clones producing E protein-specific antibodies reactive against both POWV EDIII protein and POW-VLPs (Figures 6 and 7). Such monoclonal antibodies represent potential candidates for therapeutic intervention against POWV infection. In conclusion, our study describes a novel POW-VLP vaccine candidate able to induce antibodies against POWV, which correlated with high neutralization activity. This outcome holds potential for the development of a safe and efficacious VLP-based POWV vaccine.

\section{Materials and Methods}

\subsection{Generation of POWV EDIII Recombinant Protein}

A recombinant form of the truncated Envelope Domain III (EDIII) protein of the POWV LB strain (GenPept: NP_775516) was produced by the Komagataella phaffii (Pichia pastoris) expression system and purified using nickel affinity chromatography. POWV EDIII contains residues 301 to 401 of the EDIII protein and features a thrombin cleavage site and C-terminal octa-histidine tag (BEI Resources, Manassas, VA, USA) (Cat. NR-52391).

\subsection{Production of POW-VLPS}

A plasmid construct expressing POW-VLP was designed by ATCC and was synthesized by ATUM (Newark, CA, USA). The backbone vector provided the CMV transcription promoter (Cat. pD2529-CMV-03), and the gene cassette was designed to express the prM-E gene from the POWV LB strain (GenBank NC_003687, amino acid 111-775) [36]. The POW-VLP were produced by transfection of a prM-E construct in an early passage cell culture of 293T (ATCC ${ }^{\circledR}$, Manassas, VA, USA) (Cat. CRL-3216 ${ }^{\mathrm{TM}}$ ) using Lipofectamine 3000 transfection reagent (Thermo Fisher Scientific, Waltham, MA, USA) (Cat. L3000001) following the manufacturer's instructions. The culture media consisted of DMEM culture media (ATCC ${ }^{\circledR}$, Manassas, VA, USA) (Cat. 30-2002 ${ }^{\mathrm{TM}}$ ) supplemented with $10 \%$ fetal bovine serum (FBS) $\left(\mathrm{ATCC}^{\circledR}\right.$, Cat. $\left.30-2020^{\mathrm{TM}}\right)$. After $24 \mathrm{~h}$ of the transfection, the culture media was replaced. Cells were harvested $72 \mathrm{~h}$ after transfection using a mammalian cell lysis kit (Sigma-Aldrich, St. Louis, MO, USA) (Cat. MCL-1) following the manufacturer's instructions and omitting SDS in the lysis buffer. Cell lysates were clarified by centrifugation for $30 \mathrm{~min}$ at $10,000 \times g$. VLPs were concentrated by ultracentrifugation for $2 \mathrm{~h}$ at $140,000 \times g$ with a $20 \%$ sucrose cushion in phosphate-buffered saline (PBS). Pellets were resuspended in PBS. VLPs were subsequently purified by ultracentrifugation through a continuous sucrose gradient $(10 \%$ e $50 \%$ ) for $4 \mathrm{~h}$ at $180,000 \times g$. VLPs were collected from the $20 \%$ and $30 \%$ fractions and further purified by ultrafiltration using Amicon ultra-15 filter units with a $100 \mathrm{kDa}$ cutoff (Millipore, Burlington, MA, USA) (Cat. UFC910008). Cell cultures were incubated at $37^{\circ} \mathrm{C}, 95 \%$ humidity, and $5 \% \mathrm{CO}_{2}$ in all studies. 


\subsection{Immunofluorescence Method}

VLP production in mammalian cell culture was detected by immunofluorescence assay (IFA) on the transfected cells. Cells, 293T (ATCC ${ }^{\circledR}$, Manassas, VA, USA) (Cat. CRL-3216 ${ }^{\mathrm{TM}}$ ), were plated in chamber slides (Thermo Fisher Scientific, Waltham, MA, USA) (Nunc LabTek Chamber Slide) and, after $24 \mathrm{~h}$, were transfected with POW-VLP construct as described above. A total of $48 \mathrm{~h}$ after the transfection, the cells were fixed with ice-cold methanol for $10 \mathrm{~min}$ and washed thrice with PBS. The slides were incubated for $1 \mathrm{~h}$ with a blocking buffer (3\% non-fat dry milk in PBS). After blocking, the slides were washed thrice with PBS and incubated with antibody Powassan virus immune ascitic fluid [V-518-711-562] (ATCC ${ }^{\circledR}$, Cat. VR-1262AFTM), diluted to 1:100 for $1 \mathrm{~h}$. The slides were washed thrice and incubated for $1 \mathrm{~h}$ with goat anti-mouse IgG antibody, FITC conjugate (Millipore, Burlington, MA, USA) (Cat. 5008). After washing the slides thrice with PBS, Vectashield mounting media containing DAPI for nuclear staining (Vector Laboratories, Burlingame, CA, USA) (Cat. H-1200-10) was used for preparing the slides for the epifluorescence microscopy. Cell cultures were incubated at $37^{\circ} \mathrm{C}, 95 \%$ humidity, and $5 \% \mathrm{CO}_{2}$ in all studies.

\subsection{Dot Blot}

Antigen concentration of the POW-VLP vaccine was assayed using dot blot analysis as described before [37]. Using a volumetric range of 1 to $5 \mu \mathrm{L}$, VLP samples were absorbed on nitrocellulose for $15 \mathrm{~min}$ and blocked for $1 \mathrm{~h}$ with a blocking buffer consisting of $3 \%$ non-fat dry milk in PBS containing $0.05 \%$ Tween 20 (PBS-Tween). A standard curve was obtained using recombinant Powassan virus LB EDIII protein containing a C-terminal histidine tag (BEI Resources, Manassas, VA, USA) (Cat. NR-52391). The dot blot membrane was incubated for $2 \mathrm{~h}$ with primary antibody Powassan virus immune ascitic fluid [V-518-711562] (ATCC ${ }^{\circledR}$, Manassas, VA, USA) (Cat. VR-1262AFTM), diluted in blocking buffer 1:250. After washing in PBS-tween, the membrane was incubated for $1 \mathrm{~h}$ with secondary antibody solution, goat anti-mouse IgG antibody, HRP Conjugate (ImmunoReagents, Raleigh, NC, USA) (Cat. GtxMu-003-DHRPX) diluted in blocking buffer 1:5000. After a final washing with PBS-tween, the detection was performed using enhanced chemiluminescence (ECL) substrate Radiance Q (Azure Biosystems, Dublin, CA, USA) and a digital imaging system (Azure Biosystems c600). Antigen quantification was performed using ImageJ imaging software (NIH, Bethesda, MD, USA).

\subsection{Western Blot}

Western blot was performed using Bolt 4-12\% Bis-Tris Plus gels, 10-wells (Thermo Fisher Scientific, Waltham, MA, USA) (NW04120BOX) following the manufacturer's instructions. Primary and secondary antibodies used for the immunoassays included Powassan virus immune ascitic fluid [V-518-711-562] (ATCC ${ }^{\circledR}$, Manassas, VA, USA) (Cat. VR1262 $\mathrm{AF}^{\mathrm{TM}}$ ), monoclonal anti-Langat virus envelope glycoprotein $(\mathrm{E})$, clone 10F6 (BEI Resources, Manassas, VA, USA) (Cat. NR-40316), Powassan virus prM protein antibody (GeneTex, Irvine, CA, USA) (Cat. GTX132055), His-Tag antibody (H-3) (Santa Cruz Biotechnology, Dallas, TX, USA) (Cat. sc-8036), goat anti-mouse IgG antibody, and HRP conjugate (ImmunoReagents, Raleigh, NC, USA) (Cat. GtxMu-003-DHRPX). Detection was performed using enhanced chemiluminescence (ECL) substrate Radiance $Q$ (Azure Biosystems, Dublin, CA, USA) and a digital imaging system (Azure Biosystems c600). Recombinant protein Zika Virus EDIII His-Tag was used as a protein standard (Sino Biological US, Wayne, PA, USA) (Cat. 40543-V08Y).

\subsection{Electron Microscopy}

VLP preparations were negatively stained and examined by transmission electron microscopy (TEM) at the George Washington University Nanofabrication Imaging Center, Washington, DC. Suspended particles $(10 \mu \mathrm{L})$ were applied to a glow discharged Formvar carbon-coated copper grid and incubated for $1 \mathrm{~min}$. The VLPs were fixed on grids with $4 \%$ glutaraldehyde for $5 \mathrm{~min}$ followed by water washes. The samples were then stained with 
$2 \%$ aqueous uranyl acetate for $1 \mathrm{~min}$. The grids were examined at $80 \mathrm{kV}$ using an FEI Talos F200X TEM (Thermo Fisher Scientific, Waltham, MA, USA).

\subsection{Murine Model for POW-VLP Immunization}

BALB/c mice (Mus musculus), 6-week-old males and females (Envigo, Frederick, MD, USA) were immunized by intramuscular (i.m.) injections of $50 \mu \mathrm{L}$ of POW-VLP vaccine or a placebo control. Each experimental group consisted of 5 females and 5 males. Immunizations were administered on days 1, 21 and 42, and each VLP vaccine dose contained POW-VLPs admixed in a 1:1 volume with AddaVax, a squalene-based oil-inwater nano-emulsion (InvivoGen, San Diego, CA, USA) (Cat. vac-adx-10). The placebo group received PBS admixed with AddaVax at a 1:1 volume. Serum was collected by tail vein bleeding before and after immunizations and retro-orbital bleeding at the end of the study.

\subsection{ELISA}

Serum antibody response to POW-VLP immunization was analyzed by direct ELISA, using individual serum samples. Each well of the ELISA plates Costar (Corning Inc., Corning, NY, USA) was coated with 25 ng of recombinant Envelope Domain III (EDIII) protein from the Powassan virus (BEI Resources, Manassas, VA, USA) (Cat. NR-52391) diluted in PBS and incubated at $4{ }^{\circ} \mathrm{C}$ overnight. Serum samples were serially diluted in blocking buffer ( $3 \%$ non-fat dry milk in PBS-Tween), applied in triplicate to the coated ELISA plates, and incubated for $2 \mathrm{~h}$ at room temperature. Following washes with PBSTween (PBS plus 0.05\% Tween-20), the detection was carried out using HRP conjugated anti-mouse antibodies recognizing IgG or IgG subtypes (Bio-Rad Laboratories, Hercules, CA, USA), diluted 1:10,000 in a blocking buffer, and applied to the ELISA plates for 1 $h$. After a final washing with PBS-tween, the detection was performed using enhanced chemiluminescence (ECL) substrate Radiance Q (Azure Biosystems, Dublin, CA, USA). and a microplate reader with a luminescence detection system EnVision (PerkinElmer, Waltham, MA, USA). Antibody titer was calculated using a serial dilution of serum, and the endpoint titer was defined as the reciprocal of the highest serum dilution that gives a reading above the cutoff value [38]. The cutoff value was calculated as follows: cutoff $=$ mean negative control + (3 times standard deviation) [39].

\subsection{Neutralization Assay}

The neutralization assay was performed using the reporter viral particle system established by the Pierson laboratory [26] and plaque reduction neutralization test (PRNT). An early passage cell culture of 293 (ATCC $^{\circledR}$, Manassas, VA, USA) (Cat. CRL-3216 ${ }^{\mathrm{TM}}$ ) was plated into a 6 well-plate using about $0.3 \times 10^{6}$ cells in $2 \mathrm{~mL}$ of DMEM culture media (ATCC ${ }^{\circledR}$, Cat. $30-2002^{\mathrm{TM}}$ ) supplemented with $10 \%$ fetal bovine serum (FBS) (ATCC ${ }^{\circledR}$, Cat. $\left.30-2020^{\mathrm{TM}}\right)$, a day before transfection. Transfection was performed using $500 \mu \mathrm{L}$ of OptiMEM (Thermo Fisher Scientific, Waltham, MA, USA), $1 \mu \mathrm{g}$ of replicon plasmid pWNVII-Rep-G-Z, $3 \mu \mathrm{g}$ of packaging plasmid POWV C-prM-E, and transfection reagent Lipofectamine 3000 (Thermo Fisher Scientific, Waltham, MA, USA) (Cat. L3000001) following the manufacturer's instructions. The transfection reaction was incubated for 30 min at room temperature and added to the culture dropwise. The cell media was changed after $24 \mathrm{~h}$, and reporter viral particles were harvested 2 days after transfection. Titration of reporter viral particles was achieved by inoculation to a confluent monolayer of Vero cells (ATCC ${ }^{\circledR}$, Cat. CCL-81 ${ }^{\mathrm{TM}}$ ) growing in 24 well-plate using EMEM culture media (ATCC ${ }^{\circledR}$, Cat. 30-2003 ${ }^{\mathrm{TM}}$ ) supplemented with $2 \%$ FBS $\left(\mathrm{ATCC}^{\circledR}\right.$, Cat. 30-2020 ${ }^{\mathrm{TM}}$ ). Green fluorescence protein (GFP) foci were counted using a fluorescence microscope 2-3 days following inoculation. Neutralization activity was tested by mixing a constant amount of reporter viral particles and serial dilutions of immunized mouse serum. The neutralization reactions were incubated for $1 \mathrm{~h}$ at $37^{\circ} \mathrm{C}$ before inoculation to Vero cells. The $50 \%$ inhibition by the plaque reduction neutralization test $\left(\mathrm{PRNT}_{50}\right)$ was calculated by dose-response curve 
analysis GraphPad Prism 8 (GraphPad Software, San Diego, CA, USA). Cell cultures were incubated at $37{ }^{\circ} \mathrm{C}, 95 \%$ humidity, and $5 \% \mathrm{CO}_{2}$ in all studies.

\subsection{Generation of Hybridomas Expressing Monoclonal Antibodies}

Hybridomas were established using a spleen from a mouse immunized with a high dose of POW-VLP $(10 \mu \mathrm{g})$ following the vaccination protocol described above. The hybridoma generation was obtained using the ClonaCell-HY hybridoma kit (Stemcell Technologies, Vancouver, Canada) (Cat. \#03800) following the manufacturer's instructions and using a semi-solid medium for the initial selection of clones. Hybridoma culture supernatant secreting antibodies were screened by ELISA using POWV EDIII recombinant protein as a coating antigen as described above. Dot blot analysis was employed for testing antibodies in hybridoma supernatants, using $500 \mathrm{ng}$ of POW-VLP and equal amounts of protein from cell lysate as a negative control, using a protocol described above. Cell cultures were incubated at $37{ }^{\circ} \mathrm{C}, 95 \%$ humidity, and $5 \% \mathrm{CO}_{2}$ in all studies.

\subsection{Statistics}

Data were statistically analyzed and graphed using GraphPad Prism 8 (GraphPad Software, San Diego, CA, USA). Statistical significance was measured using Student's $t$-tests between experimental groups. Images were processed using Adobe Photoshop (Adobe).

\subsection{Ethics Statement}

The animal study was conducted according to the ARRIVE guidelines (https:// arriveguidelines.org/ accessed on 10 August 2018), and approved by the Institutional Review Board (see below). This manuscript follows ICMJE (http:/ / www.icmje.org/ accessed on 30 December 2019) and journal guidelines regarding conflict of interest and disclosures.

\section{Conclusions}

In summary, we describe the creation of a novel VLP-based vaccine candidate against POWV displaying the EDIII epitope for elicitation of highly neutralizing antibodies in vivo. This study demonstrated that POW-VLP stimulated the production of serum neutralizing antibodies that correlate with the development of strong Th1- and Th2-mediated immune responses. Furthermore, immunization with this vaccine candidate induced the generation of monoclonal antibodies against POWV EDIII protein. We anticipate that the POW-VLP vaccine will induce broad-spectrum neutralizing antibodies against EDIII within the natural virion structure and thus afford protection against Powassan neuroinvasive infection. Based on preliminary assessment in the murine model, POW-VLP represents a viable, safe, and efficacious vaccine candidate for challenge studies and preclinical evaluation.

Author Contributions: The research and manuscript contributions are as follows: Conceptualization, V.C.; methodology, V.C.; formal analysis, V.C., S.S. and E.M.; investigation, V.C., S.S., E.M., D.A.-F. and C.M.; writing - original draft preparation, V.C.; writing—review and editing, V.C., S.R. and T.T.S.; data review, supervision, project administration, V.C. All authors have read and agreed to the published version of the manuscript.

Funding: This research was supported by ATCC Internal Research and Development funds.

Institutional Review Board Statement: The Institutional Animal Care and Use Committee (IACUC) of ATCC approved the mouse study protocol. The study was performed in compliance with the approved IACUC protocol in adherence with the Office of Laboratory Animal Welfare (OLAW) guidelines and the Public Health Service (PHS) Policy on Humane Care and Use of Laboratory Animals (NIH).

Informed Consent Statement: Not applicable.

Data Availability Statement: The data presented in this study has been included in the manuscript. 
Acknowledgments: We thank Christine Brantner for providing EM analysis at the George Washington University Nanofabrication Imaging Center. We express our gratitude to Theodore C. Pierson (NIAID) for donating the reporter viral particle system and providing the neutralization assay instructions. We thank the ATCC colleagues: Randy Vines, Katie Overheim, and Heather Couch for the helpful discussions and critical review of the manuscript; Jeb Suphankij for graphical design; Joseph Leonelli for his support; Zhidong Xie and Yuxia Wang for providing the POWV EDIII recombinant protein. The following reagents were obtained through BEI Resources (Manassas, VA, USA) NIAID, NIH: Envelope Domain III (EDIII) protein from Powassan Virus, LB with C-Terminal Histidine Tag, Recombinant from Yeast, NR-52391; Monoclonal Anti-Langat Virus Envelope Glycoprotein (E), Clone 10F6 (produced in vitro), NR-40316.

Conflicts of Interest: The authors declare no conflict of interest. The funders had no role in the design of the study, in the collection, analyses, or interpretation of data, in the writing of the manuscript, or in the decision to publish the results.

\section{References}

1. Mc, L.D.; Donohue, W.L. Powassan virus: Isolation of virus from a fatal case of encephalitis. Can. Med. Assoc. J. 1959, 80, 708-711.

2. Hermance, M.E.; Thangamani, S. Powassan Virus: An Emerging Arbovirus of Public Health Concern in North America. Vector Borne Zoonotic Dis. 2017, 17, 453-462. [CrossRef]

3. Ebel, G.D. Update on Powassan virus: Emergence of a North American tick-borne flavivirus. Annu. Rev. Entomol. 2010, 55, 95-110. [CrossRef]

4. Campbell, O.; Krause, P.J. The emergence of human Powassan virus infection in North America. Ticks Tick Borne Dis. 2020, 11, 101540. [CrossRef]

5. Corrin, T.; Greig, J.; Harding, S.; Young, I.; Mascarenhas, M.; Waddell, L.A. Powassan virus, a scoping review of the global evidence. Zoonoses Public Health 2018, 65, 595-624. [CrossRef] [PubMed]

6. Kemenesi, G.; Banyai, K. Tick-Borne Flaviviruses, with a Focus on Powassan Virus. Clin. Microbiol. Rev. 2019, 32. [CrossRef]

7. Taylor, L.; Stevens, T.; Destrampe, E.M.; Brown, J.A.; McGavic, J.; Gould, C.V.; Chambers, T.V.; Kosoy, O.I.; Burkhalter, K.L.; Annambhotla, P.; et al. Powassan virus infection likely acquired through blood transfusion presenting as encephalitis in a kidney transplant recipient. Clin. Infect. Dis. 2020, 72, 1051-1054. [CrossRef] [PubMed]

8. Anderson, J.F.; Armstrong, P.M. Prevalence and genetic characterization of Powassan virus strains infecting Ixodes scapularis in Connecticut. Am. J. Trop. Med. Hyg. 2012, 87, 754-759. [CrossRef] [PubMed]

9. Bogaty, C.; Drebot, M. Powassan virus-An emerging public health concern. CMAJ 2018, 190, E472. [CrossRef] [PubMed]

10. Patel, K.M.; Johnson, J.; Zacharioudakis, I.M.; Boxerman, J.L.; Flanigan, T.P.; Reece, R.M. First confirmed case of Powassan neuroinvasive disease in Rhode Island. IDCases 2018, 12, 84-87. [CrossRef] [PubMed]

11. Boigard, H.; Alimova, A.; Martin, G.R.; Katz, A.; Gottlieb, P.; Galarza, J.M. Zika virus-like particle (VLP) based vaccine. PLoS Negl. Trop. Dis. 2017, 11, e0005608. [CrossRef] [PubMed]

12. Kubinski, M.; Beicht, J.; Gerlach, T.; Volz, A.; Sutter, G.; Rimmelzwaan, G.F. Tick-Borne Encephalitis Virus: A Quest for Better Vaccines against a Virus on the Rise. Vaccines 2020, 8, 451. [CrossRef] [PubMed]

13. Kostyuchenko, V.A.; Lim, E.X.; Zhang, S.; Fibriansah, G.; Ng, T.S.; Ooi, J.S.; Shi, J.; Lok, S.M. Structure of the thermally stable Zika virus. Nature 2016, 533, 425-428. [CrossRef]

14. Pierson, T.C.; Diamond, M.S. The continued threat of emerging flaviviruses. Nat. Microbiol. 2020, 5, 796-812. [CrossRef]

15. Fuzik, T.; Formanova, P.; Ruzek, D.; Yoshii, K.; Niedrig, M.; Plevka, P. Structure of tick-borne encephalitis virus and its neutralization by a monoclonal antibody. Nat. Commun. 2018, 9, 436. [CrossRef]

16. Matveev, A.; Matveev, L.; Stronin, O.; Baykov, I.; Emeljanova, L.; Khlusevich, Y.; Tikunova, N. Characterization of neutralizing monoclonal antibody against tick-borne encephalitis virus in vivo. Vaccine 2020, 38, 4309-4315. [CrossRef] [PubMed]

17. Stettler, K.; Beltramello, M.; Espinosa, D.A.; Graham, V.; Cassotta, A.; Bianchi, S.; Vanzetta, F.; Minola, A.; Jaconi, S.; Mele, F.; et al. Specificity, cross-reactivity, and function of antibodies elicited by Zika virus infection. Science 2016, 353, 823-826. [CrossRef] [PubMed]

18. Zhao, H.; Fernandez, E.; Dowd, K.A.; Speer, S.D.; Platt, D.J.; Gorman, M.J.; Govero, J.; Nelson, C.A.; Pierson, T.C.; Diamond, M.S.; et al. Structural Basis of Zika Virus-Specific Antibody Protection. Cell 2016, 166, 1016-1027. [CrossRef] [PubMed]

19. Cimica, V.; Galarza, J.M. Adjuvant formulations for virus-like particle (VLP) based vaccines. Clin. Immunol. 2017, 183, 99-108. [CrossRef] [PubMed]

20. Donaldson, B.; Lateef, Z.; Walker, G.F.; Young, S.L.; Ward, V.K. Virus-like particle vaccines: Immunology and formulation for clinical translation. Expert Rev. Vaccines 2018, 17, 833-849. [CrossRef]

21. Compute pI/Mw Tool. Available online: https://web.expasy.org/compute_pi/ (accessed on 20 January 2016).

22. McAuley, A.J.; Sawatsky, B.; Ksiazek, T.; Torres, M.; Korva, M.; Lotric-Furlan, S.; Avsic-Zupanc, T.; von Messling, V.; Holbrook, M.R.; Freiberg, A.N.; et al. Cross-neutralisation of viruses of the tick-borne encephalitis complex following tick-borne encephalitis vaccination and/or infection. NPJ Vaccines 2017, 2, 5. [CrossRef] 
23. Lorenz, I.C.; Kartenbeck, J.; Mezzacasa, A.; Allison, S.L.; Heinz, F.X.; Helenius, A. Intracellular assembly and secretion of recombinant subviral particles from tick-borne encephalitis virus. J. Virol. 2003, 77, 4370-4382. [CrossRef] [PubMed]

24. Leonova, G.N.; Kondratov, I.G.; Ternovoi, V.A.; Romanova, E.V.; Protopopova, E.V.; Chausov, E.V.; Pavlenko, E.V.; Ryabchikova, E.I.; Belikov, S.I.; Loktev, V.B. Characterization of Powassan viruses from Far Eastern Russia. Arch. Virol. 2009, 154, 811-820. [CrossRef] [PubMed]

25. Yu, Q.; Matkovic, E.; Reagan-Steiner, S.; Denison, A.M.; Osborn, R.; Salamat, S.M. A Fatal Case of Powassan Virus Encephalitis. J. Neuropathol. Exp. Neurol. 2020, 79, 1239-1243. [CrossRef]

26. Pierson, T.C.; Diamond, M.S.; Ahmed, A.A.; Valentine, L.E.; Davis, C.W.; Samuel, M.A.; Hanna, S.L.; Puffer, B.A.; Doms, R.W. An infectious West Nile virus that expresses a GFP reporter gene. Virology 2005, 334, 28-40. [CrossRef] [PubMed]

27. Krol, E.; Brzuska, G.; Szewczyk, B. Production and Biomedical Application of Flavivirus-like Particles. Trends Biotechnol. 2019, 37, 1202-1216. [CrossRef]

28. Zhang, N.; Li, C.; Jiang, S.; Du, L. Recent Advances in the Development of Virus-Like Particle-Based Flavivirus Vaccines. Vaccines 2020, 8, 481. [CrossRef]

29. VanBlargan, L.A.; Himansu, S.; Foreman, B.M.; Ebel, G.D.; Pierson, T.C.; Diamond, M.S. An mRNA Vaccine Protects Mice against Multiple Tick-Transmitted Flavivirus Infections. Cell Rep. 2018, 25, 3382-3392.e3. [CrossRef]

30. Choi, H.; Kudchodkar, S.B.; Ho, M.; Reuschel, E.L.; Reynolds, E.; Xu, Z.; Bordoloi, D.; Ugen, K.E.; Tebas, P.; Kim, J.; et al. A novel synthetic DNA vaccine elicits protective immune responses against Powassan virus. PLoS Negl. Trop. Dis. 2020, 14, e0008788. [CrossRef]

31. Blazevic, J.; Rouha, H.; Bradt, V.; Heinz, F.X.; Stiasny, K. Membrane Anchors of the Structural Flavivirus Proteins and Their Role in Virus Assembly. J. Virol. 2016, 90, 6365-6378. [CrossRef] [PubMed]

32. Boigard, H.; Cimica, V.; Galarza, J.M. Dengue-2 virus-like particle (VLP) based vaccine elicits the highest titers of neutralizing antibodies when produced at reduced temperature. Vaccine 2018, 36, 7728-7736. [CrossRef]

33. VanBlargan, L.A.; Errico, J.M.; Kafai, N.M.; Burgomaster, K.E.; Jethva, P.N.; Broeckel, R.M.; Meade-White, K.; Nelson, C.A.; Himansu, S.; Wang, D.; et al. Broadly neutralizing monoclonal antibodies protect against multiple tick-borne flaviviruses. J. Exp. Med. 2021, 218, e20210174. [CrossRef]

34. Agudelo, M.; Palus, M.; Keeffe, J.R.; Bianchini, F.; Svoboda, P.; Salat, J.; Peace, A.; Gazumyan, A.; Cipolla, M.; Kapoor, T.; et al. Broad and potent neutralizing human antibodies to tick-borne flaviviruses protect mice from disease. J. Exp. Med. 2021, 218, e20210236. [CrossRef] [PubMed]

35. Heinz, F.X.; Stiasny, K. Flaviviruses and their antigenic structure. J. Clin. Virol. 2012, 55, 289-295. [CrossRef] [PubMed]

36. Mandl, C.W.; Holzmann, H.; Kunz, C.; Heinz, F.X. Complete genomic sequence of Powassan virus: Evaluation of genetic elements in tick-borne versus mosquito-borne flaviviruses. Virology 1993, 194, 173-184. [CrossRef]

37. Cimica, V.; Williams, S.; Adams-Fish, D.; McMahon, C.; Narayanan, A.; Rashid, S.; Stedman, T.T. Zika Virus-Like Particle (VLP) vaccine displaying Envelope (E) protein CD loop antigen elicits protective and specific immune response in a murine model. Biochem. Biophys. Res. Commun. 2020, 529, 805-811. [CrossRef]

38. Crowther, J.R. The ELISA Guidebook; Springer Science \& Business Media: Berlin, Germany, 2000; Volume 149, pp. 1-413.

39. Classen, D.C.; Morningstar, J.M.; Shanley, J.D. Detection of antibody to murine cytomegalovirus by enzyme-linked immunosorbent and indirect immunofluorescence assays. J. Clin. Microbiol. 1987, 25, 600-604. [CrossRef] [PubMed] 